\title{
A Comparative Study of Perfusion CT and 99m'Tc-HMPAO SPECT Measurement to Assess Cerebrovascular Reserve Capacity in Patients With InTERnal Carotid Artery OCCLusion
}

\author{
S. Eicker ${ }^{1}$, B. Turowski², H.-J. Heiroth ${ }^{1}$, H.-J. Steiger ${ }^{1}$, D. Hänggi ${ }^{1}$ \\ ${ }^{1}$ Department of Neurosurgery, Heinrich-Heine-University, Düsseldorf, Germany \\ ${ }^{2}$ Department of Neuroradiology, Heinrich-Heine-University, Düsseldorf, Germany
}

\begin{abstract}
Background and purpose: Patients with internal carotid artery (ICA) occlusion can demonstrate impaired cerebral vascular reserve (CVR). The detection of CVR using single photon emission CT (SPECT) is nowadays widely accepted as a predictor in the diagnostic pathway in patients considered for cerebral revascularization. Recently perfusion CT (PCT) gained widely acceptance in stroke imaging. The present study was aimed at comparing the results of perfusion CT (PCT) and ${ }^{99 \mathrm{~m} T c-H M P A O}$ SPECT with acetazolamide challenge in patients with ICA occlusion.

Methods: 13 patients were included in the prospective evaluation. Both PCT and $99 \mathrm{~m}$ Tc-HMPAO SPECT were performed before and after the administration of acetazolamide. In detail, regional cerebral blood flow $(\mathrm{rCBF})$, regional cerebral blood volume (rCBV), adapted time to peak (Tmax) and mean transit times (MTT) were compared with SPECT data.

Results: ${ }^{99 \mathrm{~m} T c-H M P A O ~ S P E C T ~ d e m o n s t r a t e d ~ a n ~ i m-~}$ pairment of CVR in six patients. A preserved CVR was present in seven patients. All patients with impaired CVR proven by SPECT had a delayed MT'T (mean $+2.98 \mathrm{~s}$ ) and a delayed Tmax (mean $+5.9 \mathrm{~s})$, (both $\mathrm{p}<0.005$ compared with the non occluded side). $66 \%$ of patients with impaired CVR in SPECT showed a complete correlation of Tmax measurements in PCT with a high positive predictive value (PPV: 88.8\%).

Conclusion: The prospective study demonstrated a highly significant correlation of perfusion parameters as detected by ${ }^{99 \mathrm{~m}}$ Tc-HMPAO SPECT and the Tmax as detected by PCT in patients with ICA occlusion. Therefore this easy-to-perform technique seems to be an adequate method for the evaluation of cerebral perfusion in patients with ICA occlusion.
\end{abstract}

Key words: cerebral vascular reserve, perfusion CT, SPECT, internal carotid artery occlusion

\section{INTRODUCTION}

Patients suffering from internal carotid artery (ICA) occlusive disease can show a remarkable but individually variable risk of subsequent stroke. The annual risk of stroke was estimated at 5.5\% per year and accentuated in patients with additional compromised cerebral blood flow [1, 21]. Therefore, besides clinical symptoms the impairment of the cerebral vascular reserve (CVR), which reflects the capacity to maintain adequate blood flow in the face of decreased perfusion pressure, has been identified as the major predictive indicator for the risk of subsequent cerebral infarction [1]. Since improvement of CVR can be achieved by extra-intracranial (EC-IC) bypass surgery, it is important to identify patients with perfusion impairment [2]. In recent years several techniques have been established to determine CVR comprising single photon emission computed tomography (SPECT) [3], transcranial Doppler (TCD) ultrasound [4], perfusion magnetic resonance imaging (MRI) [5-7], positron emission tomography (PET) [8] and Xenon computed tomography (CT) [9]. Nowadays SPECT analysis with and without acetazolamide $\left(\mathrm{Diamox}^{\circledR}\right)$ challenge is widely regarded as gold standard in the diagnostic pathway of patients with ICA occlusive disease and assumed reduction of CVR. Nonetheless, this examination has some disadvantages: First, due to tracer kinetics it has to be performed on two days [14]. Second, the analysis of the examination must be regarded as semiquantitative [6]. Finally, SPECT provides less morphological information compared to MRI and to regular CT. The goal of the present study was to correlate perfusion CT (PCT) examinations in patients with ICA occlusion with $99 \mathrm{~m}$ Tc-HMPAO SPECT in the evaluation of the cerebral CVR. To the best of our knowledge a comparison of PCT and ${ }^{99 \mathrm{~m}} \mathrm{Tc}-\mathrm{HMPAO}$ SPECT as presented in the study has hereby been performed for the first time.

\section{Methods \\ Patient Population}

Patients suffering from symptomatic arteriosclerotic ICA occlusion and Moyamoya disease considered for cerebral revascularization were included in this prospective single center study. Criteria for exclusion were age under 18 years and a time interval between PCT and $99 \mathrm{~m}$ Tc-HMPAO SPECT of longer than one week. All patients had given informed consent. 


\section{PCT PROTOCOL}

PCT was performed using a multislice CT Scanner (Volume Zoom, Siemens Erlangen/Germany, 80,0 kV, $120 \mathrm{mAs}, 1$ scan per second, over-all duration $35 \mathrm{~s}$ ). Patients were examined before and after acetazolamide application $\left(\right.$ Diamox $^{\circledR}, 1000 \mathrm{mg}$ i.v. over $2 \mathrm{~min}$ ) the same day. For each examination patients received a single bolus of $30 \mathrm{ml}$ contrast agent intravenously (400 $\mathrm{mg}$ iodine $/ \mathrm{ml}$ ) equalizing a total iodine dose of $12 \mathrm{~g}$. Two slices with a slice thickness of $10 \mathrm{~mm}$ representing all three supratentorial vascular territories were obtained. Raw data was transferred to a workstation for further processing. Analysis comprising determination of mean transit time (MTT), regional cerebral blood flow $(\mathrm{rCBF})$, regional cerebral blood volume (rCBV) and adapted time to peak (Tmax) was carried out by STROKETOOL-CT software (Version 2.0) [10]. Compared to time to peak (TTP)-based measurements Tmax analysis allows minimizing the influence of extracerebral conditions such as circulation time [11]. Perfusion maps were further evaluated by use of the computer software Angiotux CT 2D [11] as previously described. For each hemisphere the mean values of all four perfusion parameters in 18 overlapping defined cortical segments (equalizing sections of $10^{\circ}$ intersection $2^{\circ}$ ) were determined. All segments were related to vascular territories: An occipital $30^{\circ}$ segment corresponds to the vascular territory of the posterior cerebral artery, the adjacent $20^{\circ}$ section to the posterior border zone. The following $90^{\circ}$ segment correspond to the vascular territory of the middle cerebral artery, $20^{\circ}$ to the anterior border zone and the vascular territory of the anterior cerebral artery each. Data were displayed as a so-called angle plot with the anatomical localization on the $\mathrm{x}$ coordinate and the perfusion parameters on the y coordinate and as mean values of each cerebral hemisphere. The resulting diagram allowed comparing perfusion parameters before and after acetazolamide stress. CVR calculation was carried out in two different ways: On the one hand on the basis of $\mathrm{rCBF}$ data by use of the following formula: increase $(\%)=[$ post-rCBF $)-($ pre-rCBF $)] /($ pre$\mathrm{rCBF}) \times 100 \%$ whereas pre- and post-data refer to acetazolamide administration [6]. A difference in $\mathrm{CBF}$ between the occluded and non-occluded side of more than $10 \%$ was defined as impaired CVR (according to SPECT evaluations). On the other hand CVR estimation was performed on the basis of delayed MTT and Tmax. An increasing fraction of Tmax and MTT-values above a threshold of $3.5 \mathrm{~s}$ after acetazolamide indicated depletion of CVR.

\section{9m'Tc-HMPAO SPECT IMAGING PROTOCOL}

All patients were examined with the identical 2-day protocol with a maximum of one day between the two examinations. Measurement of brain perfusion was performed using stabilized exametazime (99m Tc-HMPAO) and SPECT. Image acquisition was initiated $20 \mathrm{~min}$ after injection of about $536 \mathrm{MBq}$ (range 500-670 MBq) stabilized exametazime. Acetazolamide was intravenously administered over 2 minutes during the stress examination. $15 \mathrm{~min}$ after acetazolamide infusion
99m'Tc-HMPAO was slowly injected. Patients were examined in a supine position with the head in head holders. For image acquisition, a Philips Prism 2000 XP double-head gamma camera (Marconi plc, London, England) was used. Further evaluation was carried out once the data had been transferred to a workstation. Analysis of all studies was carried out by visual interpretation and by comparison of ROIs in both hemispheres. Impairment of CVR in ${ }^{99 \mathrm{~m} T c-H M P A O}$ SPECT imaging was defined as a $\mathrm{rCBF}$ restriction of more than $10 \%$ in the occluded side compared to the non-occluded side. In reverse a difference in $\mathrm{rCBF}$ less than $10 \%$ was defined as preserved CVR [6].

\section{StATISTICAL ANALYSIS}

Statistical analysis comparing the PCT data (occluded vs. non occluded side) was performed by use of a Wilcoxon rank-sum test. This test was also applied to compare the $99 \mathrm{~m}$ Tc-HMPAO SPECT data with the PCT (SPSS, Version 16.0.1, Chicago). The positive predictive value (PPV) was calculated using the formula: number of right positive values/ (number of right-positive values + number of wrong-positive values).

\section{RESULTS \\ PATIENTS}

13 patients (five males and eight females) aged between 18 and 69 years (average age: 50.3 years) were included in the evaluation. In all patients we received comparable examinations ( 7 before and 6 after bypass surgery). A total of 13 parallel examinations were performed. Symptomatic unilateral ICA occlusion (8 patients; 3 right-sided and 5 left-sided), Moyamoya disease (4 patients) and symptomatic unilateral ICA/MCA occlusion (1 patient) were enclosed. All patients had symptoms of cerebrovascular or carotid artery disease before performing an EC-IC bypass. One patient presented in our clinic after bypass surgery for a check-up. 7 patients suffered from general arterial occlusive disease. Other cerebrovascular risk factors (arterial hypertension, hypercholesterolemia and diabetes mellitus) were present in 8 patients. One patient displayed a cerebral aneurysm: a unilateral anterior cerebral artery (ACA) aneurysm.

11 EC-IC bypass surgeries were performed. One patient underwent EC-IC bypass surgery on both symptomatic sides with a time lag of three months. Five high-flow bypass surgeries and one medium-flow bypass surgery with autogenetic radial artery graft, one barrel EC-IC bypass surgery (parietal and frontal branches of the superficial temporal artery) and four standard EC-IC bypass surgeries were completed in our department. In all patients a preoperative and postoperative study including cerebral angiography, CT, MRI (preoperative) and a perfusion imaging with and without stress simulation was performed. In 7 patients we received comparable examinations (99mTc-HMPAO SPECT/ PCT) before and in $6 \mathrm{pa}-$ tients after bypass surgery. PCT and ${ }^{99 \mathrm{~m} T \mathrm{~T}-\mathrm{HMPAO}}$ SPECT were usually performed within 4 days. Patients' data are summarized in Table 1. 


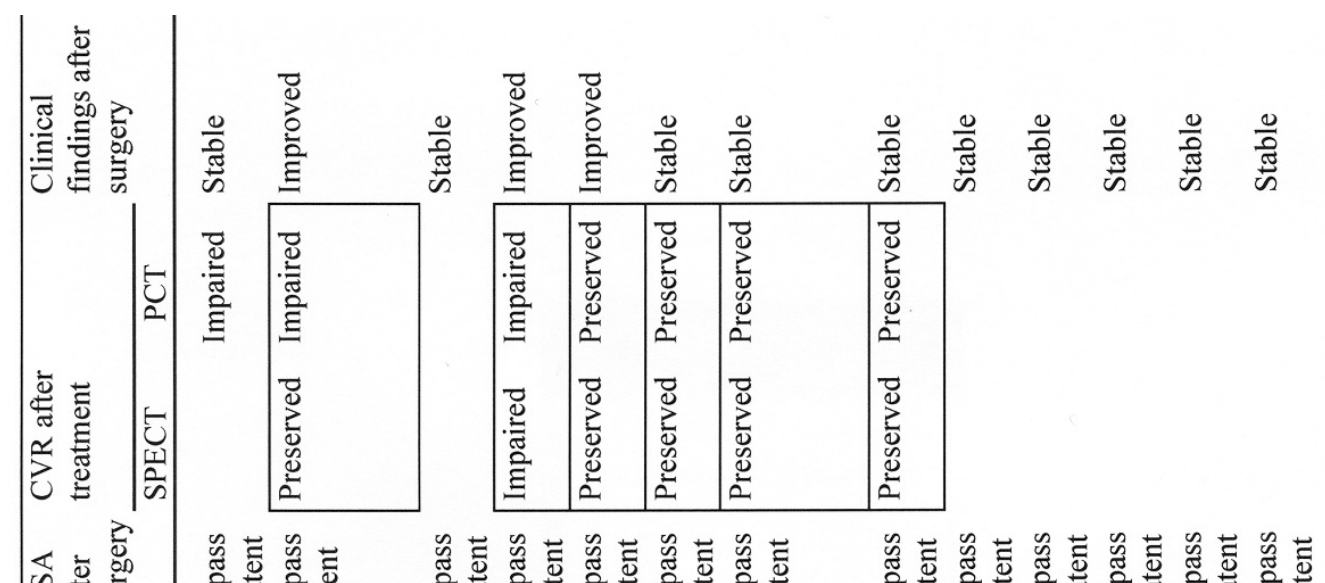

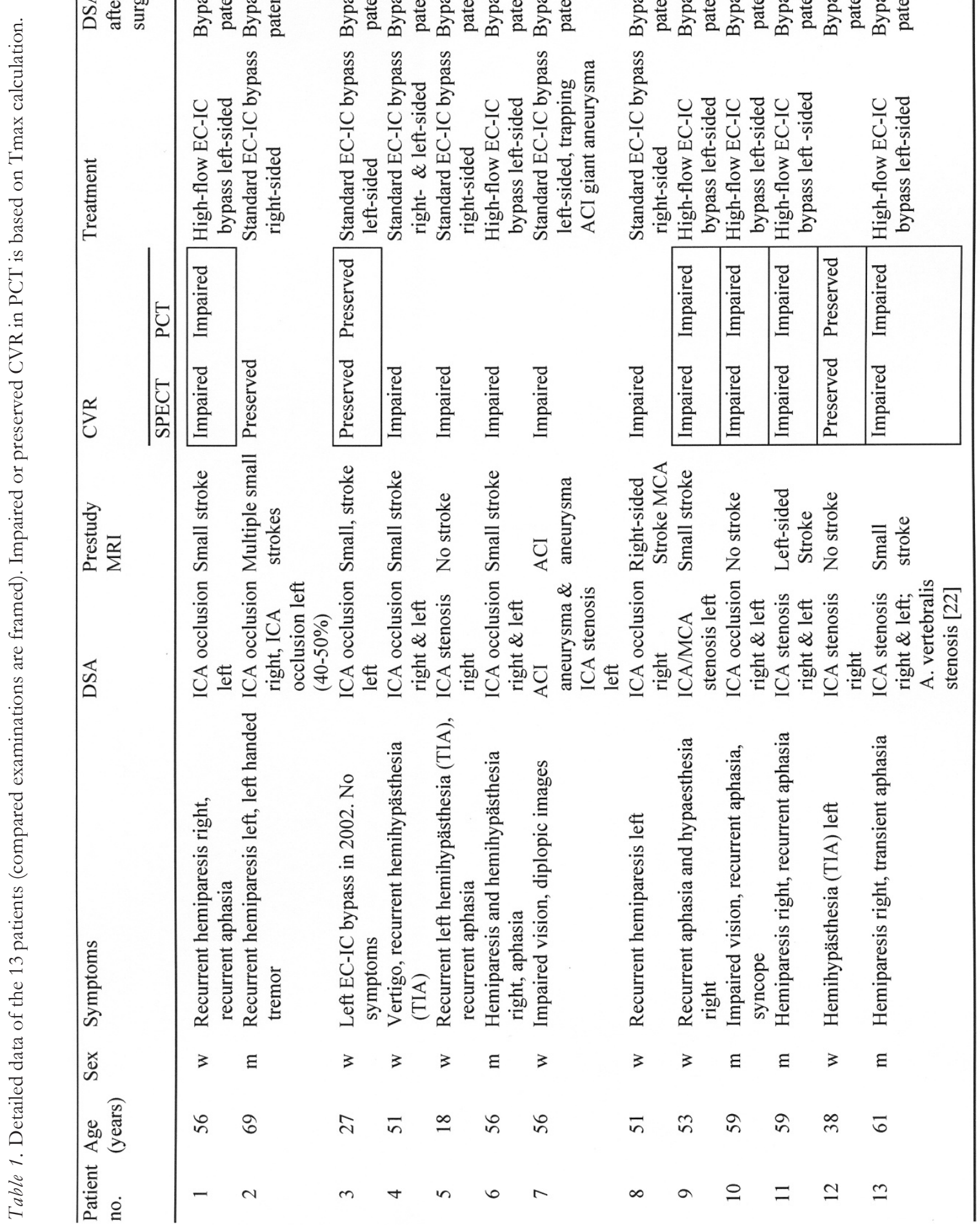




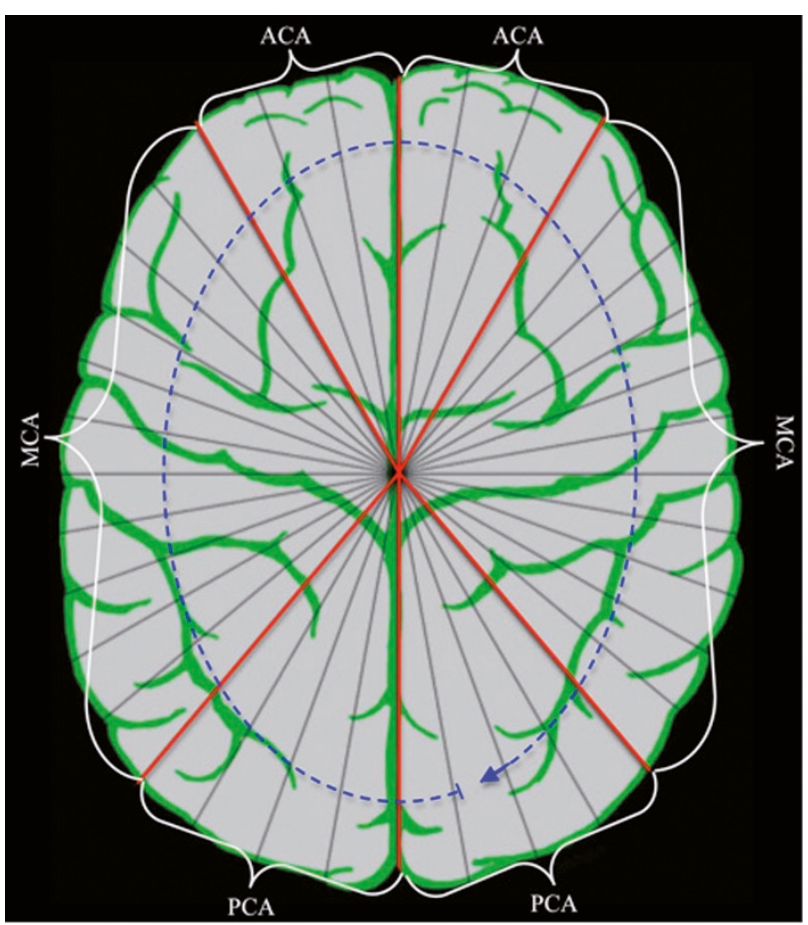

Fig. 1. Brain map devided into 36 segments and the six analysed vascular terretories.

\section{9m'TC-HMPAO SPECT RESULTS}

An impairment of CVR was found by ${ }^{99 \mathrm{~m} T \mathrm{Tc}-\mathrm{HMPAO}}$ SPECT in six analyses whereas a preservation of CVR was present in seven examinations. Table 2 gives you an overview over the examined patients in detail.
Table 2. Impaired and preserved CVR in $99 \mathrm{~m} \mathrm{Tc}-\mathrm{HMPAO}$ SPECT analyses.

\begin{tabular}{lll}
\hline Impaired CVR in SPECT & \multicolumn{2}{c}{ Preserved CVR in SPECT } \\
\hline - Unilateral ICA occlusion & 1 - Unilateral ICA occlusion & 3 \\
- Moyamoya disease & 1 - Moyamoya disease & 3 \\
- Bilateral ICA occlusion * & 3 - Bilateral ICA occlusion * & 1 \\
- ICA/MCA occlusion & 1 - ICA/MCA occlusion & -
\end{tabular}

* One symptomatic hemisphere

\section{RCBF AND rCBV RESULTS}

Four patients with unilateral ICA occlusion showed a decrease of $\mathrm{rCBF}$ in the PCT analysis on the occluded side without significant difference to the non-occluded side. One patient with unilateral ICA occlusion and reduced CVR in $99 \mathrm{~m}$ Tc-HMPAO SPECT displayed a $49.6 \%$ increase of $\mathrm{rCBF}$ in PCT on the occluded side (an asymptomatic stenosis on the contralateral side was known). One patient with ICA occlusion of the right side and asymptomatic carotid artery stenosis on the left and preserved CVR in ${ }^{99} \mathrm{~m} T \mathrm{~T}-\mathrm{HMPAO}$ SPECT presented with a $202.2 \%$ decrement in $\mathrm{rCBF}$ on the right hemisphere in comparison to the contralateral side.

In the subgroup of Moyamoya disease one patient displayed an impairment of CVR in SPECT. This patient presented with an incremented $\mathrm{rCBF}$ of the symptomatic hemisphere, without statistical significance in comparison to the contra lateral side. Two pa-
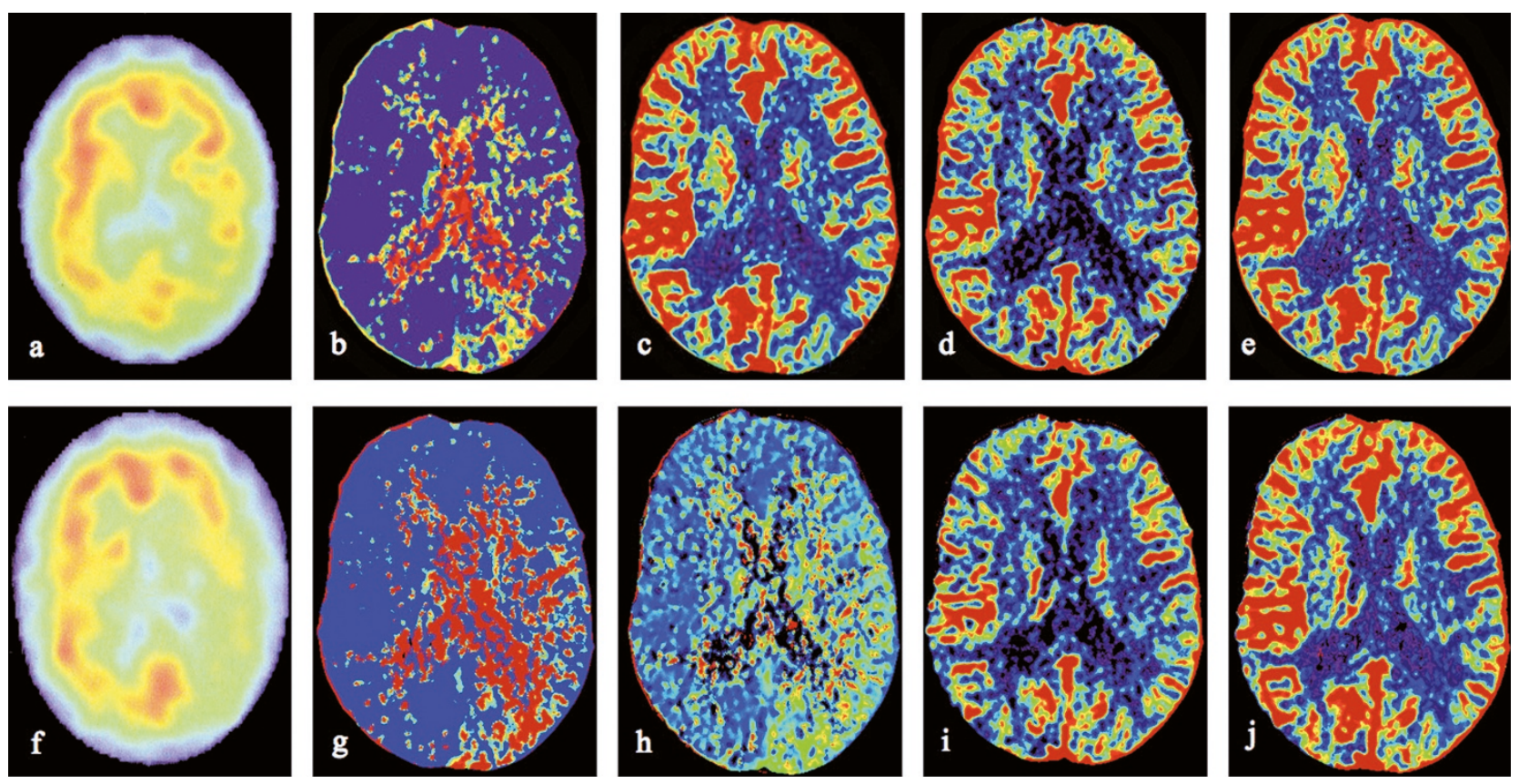

Fig. 2. Patient no. 1 (further details see Table 1) with left sided ICA occlusion. Significant impairment of CVR can be detected by ${ }^{99 \mathrm{~m} T \mathrm{~T}-H M P A O}$ SPECT. a-e show ${ }^{99 \mathrm{~m}} \mathrm{Tc}-\mathrm{HMPAO}$ SPECT and PCT images before acetazolamide administration. $\mathrm{f}-\mathrm{j}$ illustrates $99 \mathrm{~m}$ Tc-HMPAO SPECT and PCT images after acetazolamide administration. The ${ }^{99 \mathrm{~m} T \mathrm{~T}-H M P A O}$ SPECT images (a, f) demonstrate impairment in the left parietal and occipital lobes. Tmax (b, g) and MTT (c, h) measurements show a delay in the parietooccipital territories after acetazolamide $(\mathrm{g}, \mathrm{h})$. No significant difference before and after acetazolamide administration can be detected in the perfusion measurement regarding $\mathrm{CBV}(\mathrm{d}, \mathrm{i})$ and $\mathrm{CBF}(\mathrm{e}, \mathrm{j})$. 
tients with Moyamoya disease and with preserved CVR in $99 \mathrm{~m}$ Tc-HMPAO SPECT showed completely different results of rCBF: one presented with an increase by $86 \%$ and another with a decrease by $60.6 \%$ (comparison of the occluded and the less or non-occluded side). The patient with ICA/MCA occlusion presented with an incremented $\mathrm{rCBF}$, which was not statistically significant compared to the non-occluded side.

RCBV measurements also showed heterogeneous results similar to the $\mathrm{rCBF}$ measurements.

CVR calculation on the basis of $\mathrm{rCBF}$ provided the following results:

Five of the six patients with impaired CVR in 99m'Tc-HMPAO SPECT also had impaired CVR in the PCT studies. One patient without correlation (preserved CVR in PCT regarding $\mathrm{rCBF}$ ) showed symptomatic ICA/MCA occlusion. Five patients with preserved CVR in ${ }^{99 \mathrm{~m} T c-H M P A O ~ S P E C T}$ imaging were detected in the rCBF imaging with an impaired CVR (two patients with Moyamoya, one with bilateral ICA occlusion and two patients with unilateral ICA occlusion). Therefore, no significant correlation of SPECT and PCT data could be detected in the group with preserved CVR.

\section{MTT And Tmax REsults}

An increasing fraction of MT'T-values above a threshold of $3.5 \mathrm{~s}$ after acetazolamide was defined as impaired CVR.

According to our definition, a CVR was seen as preserved if more than $50 \%$ of regions of interest (ROIs) were below this critical value. For better comparison with ${ }^{99 \mathrm{~m} T c-H M P A O ~ S P E C T}$ imaging, the 36 cortical segments analyzed in PCT were pooled to six vascular territories; the posterior cerebral artery (PCA) territory, the MCA territory and the ACA territory bilateral. As a result, a significant correlation between Tmax and $99 \mathrm{~m}$ Tc-HMPAO SPECT was detected. This correlation was not evident in the comparison of rCBF and ${ }^{99 \mathrm{~m} T \mathrm{~T}-\mathrm{HMPAO} \text { SPECT. MTT and Tmax }}$ measurement yielded the following results. In the overall patient cohort, MT'T was delayed by $3.8 \mathrm{~s}$ (SD: 2.7) and Tmax was delayed by $11.92 \mathrm{~s}$ (SD: 7.3) on the occluded side (mean values). Patients with impaired

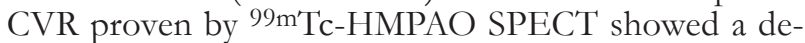
layed MTT (mean +2.98 s; SD: 2.7) and a delayed Tmax (mean +5.9 s; SD: 4.1). These findings were statistically significant with regard to the frontal and parietal vascular territories, i.e. MCA and ACA (all p< 0.005 compared with the non occluded side). However, in the posterior vascular territory we found differences that had no statistical significance.

According to our definition only Tmax values had an increasing fraction above a threshold of $3.5 \mathrm{~s}$ after acetazolamide in all subgroups witch indicated depletion of CVR.

These Tmax measurements and SPECT data showed a positive correlation. $66 \%$ of patients with impaired CVR in SPECT showed a complete consistency in Tmax measurements in the analyzed six territories in PCT $(p=1)$. In six of the remaining patients we found an absolute correlation in five of six territo- ries. Only one patient had a positive matching just in three of six territories. The precision rate for Tmax was high $(88.8 \%)$.

\section{Discussion}

A number of different techniques to determine cerebral perfusion in patients with occlusive carotid artery disease have been described. SPECT, TCD ultrasound, perfusion MRI, PET, Xenon CT with acetazolamide or $\mathrm{CO} 2$ challenge have proven to be suitable methods in the assessment of CVR $[12,13]$. In general, SPECT is widely used as the standard technique. ${ }^{15} \mathrm{O}$-PET has been reported reliable since it provides information not only about hemodynamic but also about metabolic parameters (i.e. oxygen utilization and oxygen extraction fraction) [14]. However, this procedure is limited to capacious diagnostic facilities. SPECT with acetazolamide stress is disadvantageous that examinations have to be performed in the course of two days due to tracer kinetics (a 1-day procedure is generally possible but is associated with an increase of radiation exposure for the patient as previously described) [15]. Furthermore, SPECT can merely provide vague morphological information compared with PCT or perfusion MRI [6].

PCT studies before and after acetazolamide administration can be performed on one within 30 minutes. CT images provide more morphological details [16, 17].

PCT is well established in the diagnostic pathway of stroke [1]. Recently, this examination has been reported as a predictor for secondary cerebral infarction in patients with subarchnoidal hemorrhage (SAH) [18, 19]. Encouraged by this data we hypothized that PCT can be used for the assessment of CVR with acetazolamide challenge in an analogous way to SPECT. To our knowledge a comparative analysis of PCT and 99m Tc-HMPAO SPECT has not been performed so far. For the first time we compared PCT data with $99 \mathrm{~m}$ Tc-HMPAO SPECT data in a prospective, nonrandomized setting.

One limitation of the study is the small number of patients $(n=13)$. The second restriction is the SPECT data acquisition, which was carried out in a semiquantitative matter as opposed to the quantitative PCT data collection. Therefore the detailed PCT information had to be reduced to obtain comparable measurements. A third limitation is the heterogeneous patient collective. We evaluated patients with Moyamoya disease as well as patients with ICA occlusion and even one patient with ICA/MCA occlusion.

The present study shows a significant difference in Tmax and MTT measurements on the occluded vs. the non occluded side in the frontal and parietal region. The difference in the posterior territories was not significant and could be associated with the tentorial edge and a less precise representation of the posterior supratentorial vascular territory in the measurements. Of course it can be also attributed to a lack of stenosis in the vertebro-basilar circulation. Nonetheless, the present study shows no clear correlation of PCT and SPECT regarding the rCBF and rCBV. This can be attributed to the heterogeneous patient collective and 
the small number of patients. However, we found a considerable correlation of Tmax measurement and SPECT without a difference in the subpopulations, as opposed to other examinations in which a correlation of MT'T and SPECT was detected [6, 20]. This could be due to the modified and more precise Tmax [11] data compared to the more frequently used TTP. According to our examinations, PCT can identify impairment of CVR in patients with symptomatic carotid artery occlusion in the acetazolamide stress test and corresponds favorably to $99 \mathrm{~m}$ 'Tc-HMPAO SPECT data. In the present study, Tmax data seems to be a suitable parameter for CVR estimation using the definitions of delayed Tmax as described above (precision rate of $88.8 \%$ to identify patients with preserved CVR).

Compared to SPECT, PCT provides additional morphological and hemodynamic information. Furthermore it is more readily accessible. Consequently PCT appears to be an adequate method to identify patients with impaired CVR prior to bypass surgery, However, this remains to be supported by prospective investigations with a larger patient cohort and quantitative SPECT data.

\section{CONCLUSIONS}

The present non-randomized, prospective study demonstrated a highly significant correlation of the perfusion parameters as detected by ${ }^{99 \mathrm{~m} T \mathrm{~T}-\mathrm{HMPAO}}$ SPECT and the Tmax as detected by PCT in patients with ICA occlusion. Therefore the easy-to-perform PCT examination seems to be an adequate method for the evaluation of cerebral perfusion in patients with ICA occlusion.

Conflict of interest Statement: We declare that we have no conflict of interest.

\section{REFERENCES}

1. Yonas H, Smith HA, Durham SR, Pentheny SL, Johnsosn DW (1993). Increased stroke risk predicted by compromised cerebral blood flow reactivity. J Neurosurg 79: 48389

2. Schmiedek P, Piepgras A, Leinsinger G, Kirsch CM, Einhäupl K (1994). Improvement of cerebrovascular reserve capacity by C-IC arterial bypass surgery in patients with ICA occlusio and haemodynamic cerebral ischemia. J Neurosurg 81:236-244

3. Matsuda H, Higashi S, Kinuya K, Tsuji S, Nozaki J, Sumiya H, Hisada K, Yamashita J (1991). SPECT evaluation of brain perfusion reserve by acetazolamide test using Tc-99m HMPAO. Clin Nucl Med 16:572-79

4. Piepgras A, Schmiedek P, Leinsinger G, Haberl RL, Kirsch CM, Einhäupl KM (1990). A simple test to assess cerebrovascular reserve capacity using transcranial Doppler sonography and acetazolamide. Stroke 21:130611

5. Kim JH, Lee EJ, Lee SJ, Choi NC, Lim BH, Shin T (2002). Reliability of perfusion MR imaging in symptomatic carotid occlusive disease. Cerbral blood volume, mean transit time and time-to-peak. Acta Radiol 43:36064

6. Ma J, Mehrkens JH, Holtmannspoetter M, Linke R, Schmid-Elsaesser R, Steiger HJ, Brueckmann H, Bruening R (2007). Perfusion MRI before and after acetazo- lamide administration for assessment of cerebrovascular reserve capacity in patients with symptomatic internal carotid artery (ICA) occlusion: comparison with $99 \mathrm{mTc}$ ECD SPECT. Neuroradiology 49: 317-26

7. Kikuchi K, Murase K, Miki H, Kikuchi T, Sugawara Y, Mochizuki T, Ikezoe J, Ohue S (2001). Measurement of cerebral hemodynamics with perfusion-weighted MR imaging: comparison with pre- and postacetazolamide 133Xe-SPECT in occlusive carotid disease. AJNR Am J Neuroradiol 22: 248-54

8. Derdeyn CP, Videen TO, Simmons NR, et al (1999) Count-based PET method for predicting ischemic stroke in patients with symptomatic carotid arterial occlusion. Radiology 212:499-506

9. Furukawa M, Kashiwagi S, Matsunaga N, Suzuki M, Kishimoto K, Shirao S (2002). Evaluation of cerebral perfusion parameters measured by perfusion $\mathrm{CT}$ in chronic cerebral ischemia: comparison with xenon CT. J Comput Assit Tomogr 26:272-8

10. Wittsack HJ, Kleiser R, Cohnen M, Moedder U (2004). TPerfusion Imaging with Deconvolution Analysis by Singular Value Decomposition: Misuse your Old CT-Scanner for Cerebral Perfusion Analysis. Congress of the Radiological Society of North America RSNA, Chicago. Available via:

http://rsna2004.rsna.org/rsna2004/V2004/conference/ event_display.cfm?em_id=4410557. Accessed 2004

11. Turowski B, Haenggi D, Wittsack HJ, Beck A, Aurich V (2007). Computerized Analysis of Brain Perfusion Parameter Images. Rofo 179:525-29

12. Derdeyn CP, Grubb RL, Powers WJ (1999). Cerebral hemodynamic impairment: methods of measurement and association with stroke risk. Neurology 53:251-59

13. Haralanov L, Klissurski M, Stamenova P (2001). Functional methods for evaluation the occurrence of delayed ischemic deficit in patients with subarachnoid hemorrhage. Eur J Med Res 6:185-9

14. Kuwabara Y, Ichiya Y, Sasaki M (1998). PET evaluation of cerebral hemodynamics in occlusive cerebrovascular disease pre- and postsurgery. J Nucl Med 39:760-65.

15. Hattori N, Yonekura Y, Tanaka F, Fujita T, Wang J, Ishizu K, Okazawa H, Tamaki N, Konishi J (1996). Oneday protocol for cerebral perfusion reserve with acetazolamide. J Nucl Med 37:2057-61

16. Chen A, Shyr MH, Chen TY, Lai HY, Lin CC, Yen PS (2006). Dynamic CT perfusion imaging with acetazolamide challenge for evaluation of patients with unilateral cerebrovascular steno-occlusive disease. AJNR Am J Neuroradiol 27: 1876-81

17. Miyazawa N, Arbab AS, Umeda T, Akiyama I (2005). Perfusion CT investigation of chronic internal carotid artery occlusion: comparison with SPECT. Clin Neurol Neurosurg 108:11-7

18. Miyazawa N, Arbab AS, Umeda T, Akiyama I (2006). Vasospasm after subarachnoid hemorrhage: utility of perfusion CT and CT angiography on diagnosis and management. AJNR Am J Neuroradiol 27: 26-34

19. Pham M, Johnson A, Bartsch AJ, Lindner C, Müllges W, Roosen K, Solymosi L, Bendszus M (2007). CT perfusion predicts secondary cerebral infarction after aneurismal subarachnoidal hemorrhage. Neurology 69: 262-765

20. Kamath A, Smith WS, Powers WJ, Cianfoni a, Chien JD, Videen T, Lawton MT, Finley B, Dillon WP, Wintermark M (2008). Perfusion CT compared to H2 15O/O15O PET in patients with chronic cervical carotid artery occlusion. Neuroradiology 50:745-51

21. Klijn CJ, Kappelle LJ, Tulleken CA, van Gijn J (1997). Symptomatic carotid atery occlusion. A reappraisal of hemodynamic factors. Stroke 28:2084-93 
22. Stangel M, Lüchow A, Stapf C, Marx P, Mohr JP, Mast H (1997). Cerebral atrophy with basilar artery occlusion. Eur J Med Res 2:514

Received: March 8, 2011 / Accepted: May 5, 2011
Address for correspondence:

Dr. Sven O. Eicker

Department of Neurosurgery

Heinrich-Heine-University

Moorenstraße 5

40225-Düsseldorf

Germany

Tel.: ++49 2118116268

Fax: ++492118119556

E-mail: Sven.Eicker@med.uni-duesseldorf.de 